\title{
Metformin attenuates renal interstitial fibrosis through upregulation of Deptor in unilateral ureteral obstruction in rats
}

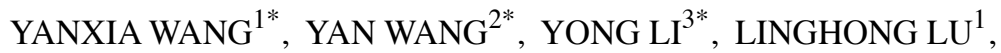 \\ YINGXIAN PENG ${ }^{1}$, SHU ZHANG ${ }^{1}$ and ANZHOU XIA ${ }^{1}$ \\ ${ }^{1}$ Department of Pharmacology, Xuzhou Medical University; ${ }^{2}$ Department of Pharmacy, \\ Affiliated Hospital of Xuzhou Medical University, Xuzhou, Jiangsu 221000; ${ }^{3}$ Department of Gastrointestinal Surgery, \\ Xuzhou Central Hospital, Xuzhou, Jiangsu 221006, P.R. China
}

Received April 16, 2019; Accepted January 14, 2020

DOI: $10.3892 / \mathrm{etm} .2020 .9144$

\begin{abstract}
Renal interstitial fibrosis (RIF) is a common pathological process that accompanies chronic kidney disease (CKD) and that progresses to end-stage renal failure (ESRD). Accumulating evidence has revealed that persistent mammalian target of rapamycin (mTOR) activation in kidneys is closely associated with the occurrence and progression of CKD. The DEP domain-containing mTOR interacting protein (Deptor) is an endogenous negative regulator of mTOR. Metformin can attenuate renal fibrosis in an animal model of diabetic nephropathy. Previous studies demonstrated that metformin can attenuate renal fibrosis in several models of CKD. However, the precise mechanisms of this effect are not well understood. The present study aimed to examine the mechanism of action of metformin on unilateral ureteral obstruction (UUO)-induced RIF in rats in vivo. Sprague-Dawley rats were randomly divided into a sham-operated group, three UUO groups examined at different time points (3,7 and 14 days after UUO surgery), and three metformin-treated groups, treated with three different concentrations of metformin. The metformin-treated groups were administered metformin orally every day for 14 consecutive days following surgery. The protein expression levels of Deptor, $\alpha$-smooth muscle actin ( $\alpha$-SMA), phosphorylated (p-)mTOR, p-ribosomal protein S6 kinase (p-p70S6K) and CD68 were assessed. The present results suggested that, following UUO, there was a significant reduction of Deptor expression, and an increase in collagen deposition in the extracellular matrix over time, accompanied by an increased expression of several proteins
\end{abstract}

Correspondence to: Mr. Anzhou Xia, Department of Pharmacology, Xuzhou Medical University, 209 Tongshan Road, Xuzhou, Jiangsu 221000, P.R. China

E-mail: xiaanzhou@xzhmu.edu.cn

*Contributed equally

Key words: metformin, DEP domain-containing mTOR interacting protein, mTOR, interstitial fibrosis, unilateral ureteral obstruction including CD68, $\alpha$-SMA, p-mTOR and p-p70S6K. Notably, metformin treatment reversed these effects. In conclusion, the present results suggested that metformin attenuated RIF of UUO rats, and the mechanism of action was found to be associated with the increase in Deptor expression and inhibition of the $\mathrm{mTOR} / \mathrm{p} 70 \mathrm{~S} 6 \mathrm{~K}$ pathway in the kidneys of UUO rats.

\section{Introduction}

Renal interstitial fibrosis (RIF) is a final common pathway for progression of all the various chronic kidney diseases (CKD) to end-stage renal disease (ESRD), causing the need of continuous renal replacement therapy in form of dialysis or transplantation (1). The main features of RIF, including renal tubular atrophy/dilatation, interstitial inflammatory cell infiltration, induction of apoptosis, fibroblasts activation and extracellular matrix (ECM) deposition, have been previously described (2). However, the antifibrotic therapeutic strategies currently available have not been able to effectively delay progression of CKD to ESRD or to inhibit the side effects associated with kidney diseases $(3,4)$. Therefore, it is of great importance to understand the mechanism underlying RIF in order to develop optimal strategies that are effective and safe for treating patients with CKD.

The mammalian target of rapamycin (mTOR), a serine/threonine protein kinase, is a member of the phosphatidylinositol-related kinase (PI3K) family and serves an important role in cell growth, proliferation and differentiation (5). Accumulating evidence demonstrated that persistent activation of mTOR is associated with the development of RIF in a rat model of hypertensive nephropathy (6), and in mice models of unilateral ureteral obstruction (UUO) (7) and polycystic nephropathy $(8,9)$. Therefore, inhibition of mTOR activity may be an effective therapeutic strategy for CKD treatment.

The activity of endogenous mTOR is not only regulated by its own expression level, but also by its antagonists. The DEP domain-containing mTOR interacting protein (Deptor) is an important component of the mTOR complex and a constitutive inhibitor of mTOR activity, acting as an endogenous negative regulator of the mTOR signaling pathway (10). Deptor is involved in several cellular processes, such as cell growth, 
apoptosis, autophagy and anti-inflammatory responses (11). Several previous studies have showed that Deptor is highly expressed in mesangial cells, proximal tubular epithelial cells and embryonic kidney cells, and it is less expressed in renal cancer cells (11-13). Moreover, accumulating evidence demonstrated that Deptor is associated with the pathogenesis of various kidney diseases (14-16). Nevertheless, to the best of our knowledge, no previous studies showed that Deptor is involved in the development of RIF caused by UUO in rat.

Metformin is a biguanide compound that has been used as an oral anti diabetic drug for $>50$ years. In addition to its glycemia-lowering effects, metformin has anti-inflammatory, anti-cancer and anti-aging effects (17). Furthermore, metformin may prevent or delay the onset and progression of renal fibrosis in mice with UUO (18). Recently, it was reported that the anti-cancer effect of metformin is associated with the inhibition of the Deptor-mTOR signaling pathway (17). However, to the best of our knowledge, whether the mechanism underlying the anti-renal interstitial fibrosis efficacy of metformin is associated with the regulation of the Deptor-mTOR signaling pathway in the kidney has not been previously studied. Therefore, the present study aimed to investigate whether the effect and mechanism of metformin against RIF involved the Deptor-mTOR signaling pathway using an experimental model of RIF in rats following UUO.

\section{Materials and methods}

Animals. Ten-week-old male Sprague-Dawley (SD) rats, (weight, 180-220 g) were purchased from the Laboratory Animal Center of Xuzhou Medical University (Xuzhou, China). The animals were housed in a 12-h light/dark cycle under controlled temperature $\left(23 \pm 1^{\circ} \mathrm{C}\right)$ and at $65-70 \%$ relative humidity. All animal experiments were approved by The Animal Ethics Committee of Xuzhou Medical University (Xuzhou, China). Every effort was made to minimize animal stress.

Animal grouping and experimental protocols. 54 SD rats were randomly divided into various groups: i) A sham-operated group (sham group); ii) three UUO groups examined at three different time points (3, 7 and 14 days after UUO); iii) a M50 group (UUO rats treated with low-dose metformin at $50 \mathrm{mg} / \mathrm{kg} /$ day); iv) a M100 group (UUO rats treated with medium-dose metformin at $100 \mathrm{mg} / \mathrm{kg} /$ day); and v) a M200 group (UUO rats treated with high-dose metformin at $200 \mathrm{mg} / \mathrm{kg} / \mathrm{day}$ ). Rats treated with metformin were treated once a day $(1 \mathrm{ml} / 100 \mathrm{~g})$ for 14 consecutive days after surgery. The rats in the sham and UUO groups were administered equal volumes of $0.5 \%$ sodium carboxymethyl cellulose (CMC-Na, vehicle). Metformin and vehicle were given via intragastric administration. In addition, metformin was administered orally to the normal rats with the high dose at $200 \mathrm{mg} / \mathrm{kg}$ simply had no influence on the weight and renal function compared with the sham group, which excluded the renal toxicity of the drug, and that was consistent with the previous research (19).

For UUO surgery, rats were anesthetized with $10 \%$ chloral hydrate $(350 \mathrm{mg} / \mathrm{kg})$ by intraperitoneal injection (i.p. injection), and the left lateral dorsal surface of the rat was incised, the left ureter was isolated and ligated with silk sutures, which were cut at the middle of the ligation sites to prevent retrograde urinary tract infections. Sham surgeries were performed in a similar manner, except for the ureter ligation. At the end of the experiment, each rat (weight, 210-250 g) was euthanized via exsanguination from the abdominal aorta (blood volume extracted, $\sim 9 \mathrm{ml}$ ) under deep anesthesia (10\% chloral hydrate, $400 \mathrm{mg} / \mathrm{kg}$, i.p.injection), and death was confirmed by checking for lack of breath and heartbeat. The kidney was removed, and one part of the kidney was fixed in $4 \%$ paraformaldehyde for subsequent histological analysis. The remaining parts of the kidneys and blood samples were stored at $-80^{\circ} \mathrm{C}$ for biochemical analysis. Sham operation-induced minor renal damage in the sham group, there was no significant change in the content of collagen and renal function within two weeks, and sample of the sham group was collected at 14 days after UUO surgery. Following intraperitoneal administration of chloral hydrate, no obvious signs and symptoms of peritonitis were observed in any animal during the course of the experiment.

Renal function assessment. The serum creatinine (Scr) was measured using a picric acid method and blood urea nitrogen (BUN) was measured using a urease assay as previously described $(20,21)$. Serum was obtained from blood samples collected in non-heparinized vacuum tubes, then centrifuged at 3,500 x g for $15 \mathrm{~min}$. Serum samples were stored at $-80^{\circ} \mathrm{C}$ for further analysis. The Scr and BUN levels of the rats were measured with Scr and BUN assay kits (Jiancheng Bioengineering Institute, Nanjing, China), respectively.

Histological analysis. Kidney tissues were fixed in $4 \%$ formaldehyde and embedded in paraffin for histological analysis. Then, 4- $\mu \mathrm{m}$-thick paraffin sections were dewaxed and hydrated. The sections were stained with a Sirius-Red staining kit (Bogoo Biotechnology Co. Ltd.) according to the manufacturer's protocol. Then, the sections were dehydrated to transparency, mounted with neutral gum and examined using an Olympus BX43F microscope (Olympus Corporation). All histopathological alterations were evaluated by two blinded investigators, five sections were randomly selected in each slide, and five randomly-selected fields of view were analyzed in each section. The percentage of positive areas were analyzed using the Image-Pro Plus 4.0 software. The kidney weight index $(\mathrm{mg} / \mathrm{g})$ was calculated as the ratio between the weight of the left or right kidney $(\mathrm{mg})$ and the body weight $(\mathrm{g})$ of the rat.

Immunohistochemistry analysis. Assays of Deptor and CD68 levels by immunohistochemistry were performed as previously described (22). The kidney sections embedded with paraffin were deparaffinized in xylene and hydrated through a decreasing series of alcohol. To inhibit endogenous peroxidase activity, sections were subsequently placed in $3 \% \mathrm{H}_{2} \mathrm{O}_{2}$. Then, the sections were blocked with $3 \%$ BSA, and incubated with anti-Deptor (1:100; Cell Signaling Technology, Inc.) and anti-CD68 (1:50; Boster) antibodies overnight at $4{ }^{\circ} \mathrm{C}$. The sections were stained using a polymer HRP detection kit (ZSGB-BIO) and counterstained with hematoxylin. An Olympus BX43F microscope (Olympus Corporation) was used to examine the sections, and the positive Deptor and CD68 signals were quantified using the integrated optical density value with the Image-Pro Plus 4.0 software. 

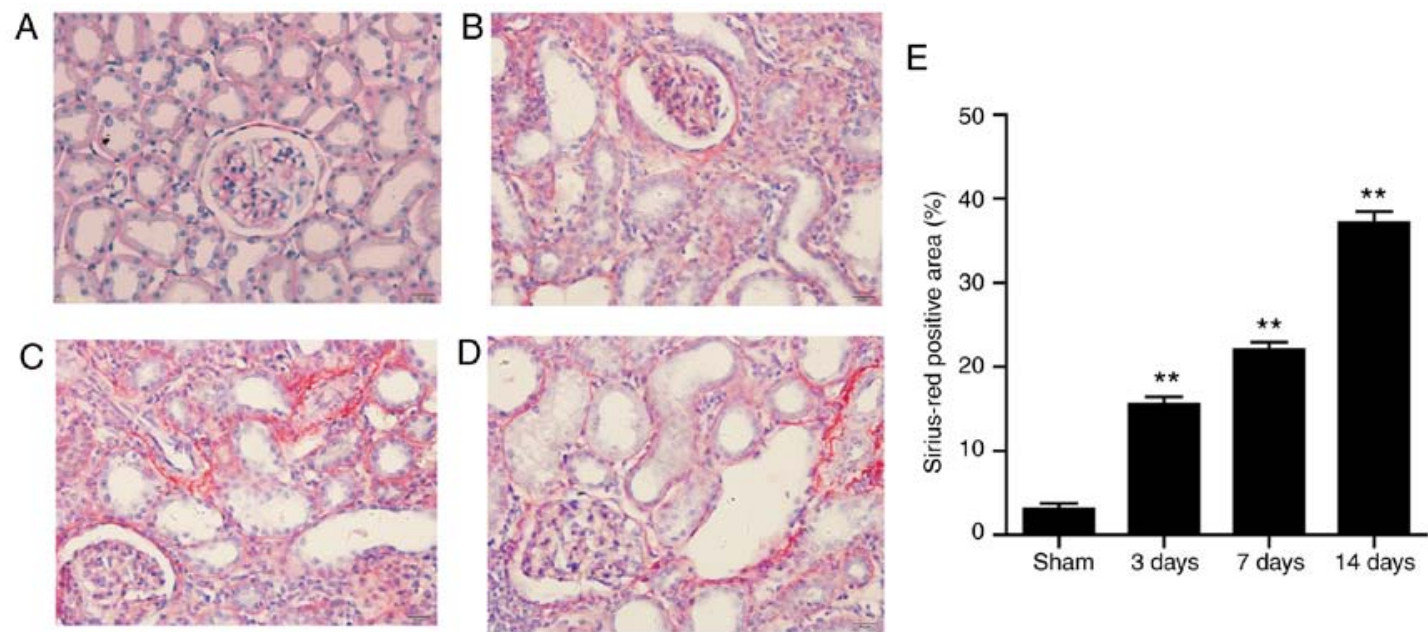

3 days 7 days 14 days

Figure 1. Expression of collagen in the progression of renal interstitial fibrosis induced by UUO. (A) Sham operation. Collagen deposition at (B) 3, (C) 7 and (D) 14 days after UUO surgery. (E) Statistical analysis of Sirius-Red positive area. Content of collagen was assessed by Sirius red-staining on sections of rat kidneys. A large amount of collagen was accumulated in the glomerular and tubular interstitial areas, which increased gradually over time. Highest level of collagen was detected at 14 days after surgery. Magnification, $\mathrm{x} 400$. All data are presented as the mean \pm SEM. $n=6$. ** $\mathrm{P}<0.01$ vs. sham group. UUO, unilateral ureteral obstruction.

Western blot analysis. Proteins were extracted from kidney tissues using RIPA lysis buffer as previously described (23). The supernatant was collected, and the concentration was determined using a bicinchoninic acid protein assay kit (Beyotime Institute of Biotechnology). Then, protein samples $(40-60 \mu \mathrm{g})$ were separated using $8-10 \%$ SDS-PAGE and transferred onto nitrocellulose membranes. The membranes were then blocked in PBS containing 3\% BSA and incubated overnight at $4^{\circ} \mathrm{C}$ with appropriate primary antibodies, including anti-mTOR (1:1,000; Cell Signaling Technology, Inc.), anti-phosphorylated (p-)mTOR (Ser 2448, 1:1,000; Cell Signaling Technology, Inc.), anti-p70S6K (1:500; Cell Signaling Technology, Inc.), anti-p-p70S6K (Ser 371, 1:500; Cell Signaling Technology, Inc.), anti-Deptor (1:800; Cell Signaling Technology, Inc.) and anti- $\alpha$-SMA (1:1,000; Abcam). Near-infrared fluorescence-conjugated secondary antibodies $(1: 10,000 ; \mathrm{LI}-\mathrm{COR})$ were used to detect the proteins at room temperature and were developed colorimetrically using the Odyssey biocolor infrared fluorescence imaging system (LI-COR). Quantification was performed by measuring the signal intensity using the ImageJ software.

Statistical analysis. The results are presented as the mean \pm SEM. Comparisons between groups were performed using one-way ANOVA followed by Tukey's test. $\mathrm{P}<0.05$ was considered to indicate a statistically significant difference. Statistical analysis was performed using SPSS 13.0 statistical software.

\section{Results}

Dynamic changes of Deptor during RIF. The amount of fibrotic tissue as measured by Sirius-Red staining. Kidneys from sham-operated rats exhibited no or very weak positive Sirius-Red staining, while large amount of fibrotic tissue was measured by Sirius-Red staining was accumulated in the glomeruli and interstitial regions of UUO rats, which increased gradually over time. Highest level of collagen was detected at 14 days after surgery (Fig. 1A-E). Immunohistochemistry staining results showed that Deptor decreased in the interstitial and glomerular mesangial area in the UUO groups (Fig. 2A-E). Western blotting results were in line with the immunohistochemistry staining results (Fig. 2F and G). Furthermore, a correlation was identified between collagen and Deptor levels. The present results suggested that collagen and Deptor levels were negatively correlated (Fig. $2 \mathrm{H}$ ).

Effects of metformin on hypertrophy of kidney and renal functions in UUO rats. Compared with the sham group, UUO rats had significantly higher left and right kidney weight index, and the left kidney weight index was increased at higher levels than that in the right kidney (Fig. 3A). Compared with the UUO group, medium and high doses of metformin significantly inhibited the increase in left kidney weight index. Low dose of metformin slightly reduced the left kidney weight of UUO rats, but the difference was not statistically significant. By contrast, the three doses of metformin did not significantly affected the right kidney weight index.

The results of renal function test showed that the levels of BUN (Fig. 3B) and Scr (Fig. 3C) in the UUO group increased significantly compared with sham rats. Compared with the UUO untreated group, low, medium and high doses of metformin significantly reduced the level of BUN in UUO rats (Fig. 3B). High and medium doses of metformin reduced the level of Scr (Fig. 3C). Low dose of metformin reduced the level of Scr, but the difference was not statistically significant (Fig. 3C).

Effects of metformin on CD68 in UUO rats. CD68 protein was lowly expressed in glomerular mesangial and renal interstitial regions in the sham group. By contrast, a high number of regions positive for CD68 staining were identified in the UUO group, indicating the presence of an increased number of infiltrating macrophages. Compared with the UUO group, three doses of metformin reduced the area positive for CD68 

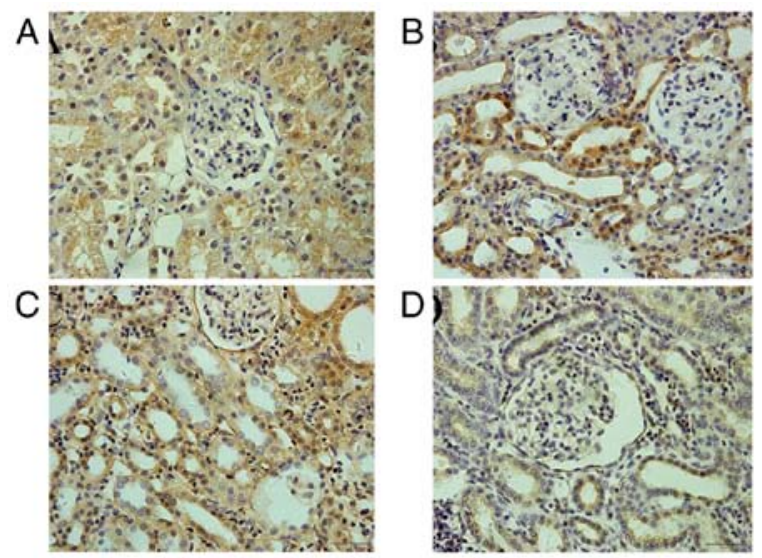

F
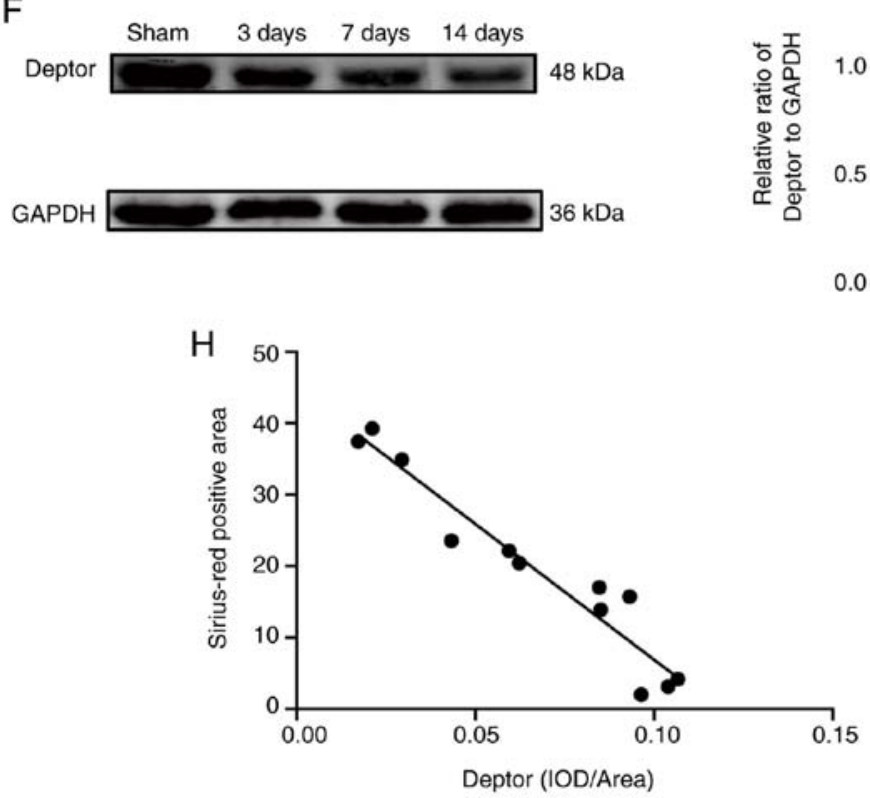
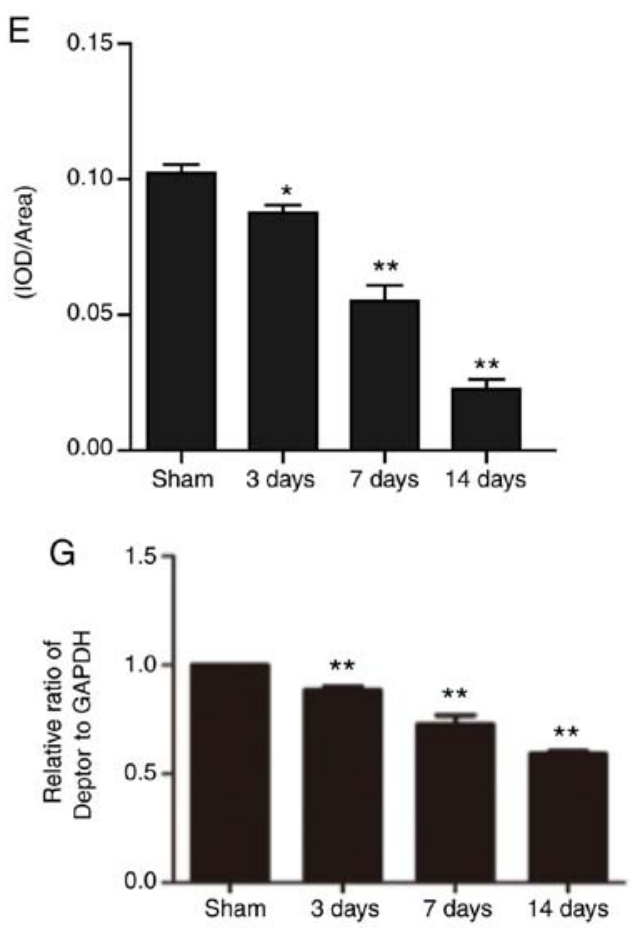

Figure 2. Dynamic changes of Deptor in the progression of renal interstitial fibrosis by immunohistochemistry. Deptor expression in kidneys of rats in the (A) sham group and in rats after (B) 3, (C) 7 and (D) 14 days after UUO. Magnification, x400 (E) Statistical analysis of Deptor immunohistochemistry positive area. $n=6$. (F) Protein expression level of Deptor in the kidneys of rats was analyzed by western blotting. (G) Statistical analysis of Deptor expression. $n=3$. (H) Correlation analysis of Deptor and collagen levels. Sirius-Red positive area of collagen and Deptor immunohistochemistry positive area were plotted and analyzed using Pearson's correlation coefficient analysis. Magnification $\mathrm{x} 400$. All data are presented as the mean $\pm \mathrm{SEM}$. ${ }^{*} \mathrm{P}<0.05$, $^{* *} \mathrm{P}<0.01 \mathrm{vs}$. sham group. UUO, unilateral ureteral obstruction; Deptor, DEP domain-containing mTOR interacting protein; IOD, integral optical density.

in the kidney $(\mathrm{P}<0.01)$, indicating that metformin reduced the infiltration of macrophages in kidney (Fig. 4A-F).

Effects of metformin on renal fibrosis in UUO rats. To observe the effect of metformin on collagen deposition, the degree of RIF in rats was examined by Sirius-Red staining. As shown in Fig. 5, collagen deposition was reduced in the sham group. However, there were a large number of red-positive areas in the UUO group, indicating that collagen deposition increased in the kidney of UUO rats. Compared with the UUO group, three doses of metformin significantly decreased the collagen deposition in renal tubules and renal interstitial regions $(\mathrm{P}<0.01)$, suggesting that metformin decreased the deposition of collagen in renal tissue of UUO rats (Fig. 5A-F).

The myofibroblast, an activated fibroblast characterized by $\alpha$-SMA positive expression, plays an important role in RIF. Therefore, the protein expression level of $\alpha$-SMA was detected by western blotting. $\alpha$-SMA protein in the kidney of normal rats was lowly expressed. However, the expression of $\alpha$-SMA protein in the kidney of UUO rats was significantly higher than that in the sham group. Low, medium and high doses of metformin reversed the protein expression level of $\alpha$-SMA in rat kidney. The present results suggested that metformin reduced the UUO-induced RIF (Fig. 5G and H).

Effects of metformin on expression of Deptor in the kidneys of UUO rats. The expression of Deptor protein was decreased during RIF and was negatively correlated with collagen expression. Therefore, the effects of metformin on Deptor protein expression were examined in renal tissue of UUO rats. Immunohistochemistry and western blotting suggested that Deptor protein was highly expressed in the kidney of rats in the sham group. Compared with the sham group, the expression of Deptor in UUO rats was significantly decreased, whereas the three doses of metformin upregulated the expression level of Deptor protein in the kidneys (Fig. 6A-H). 

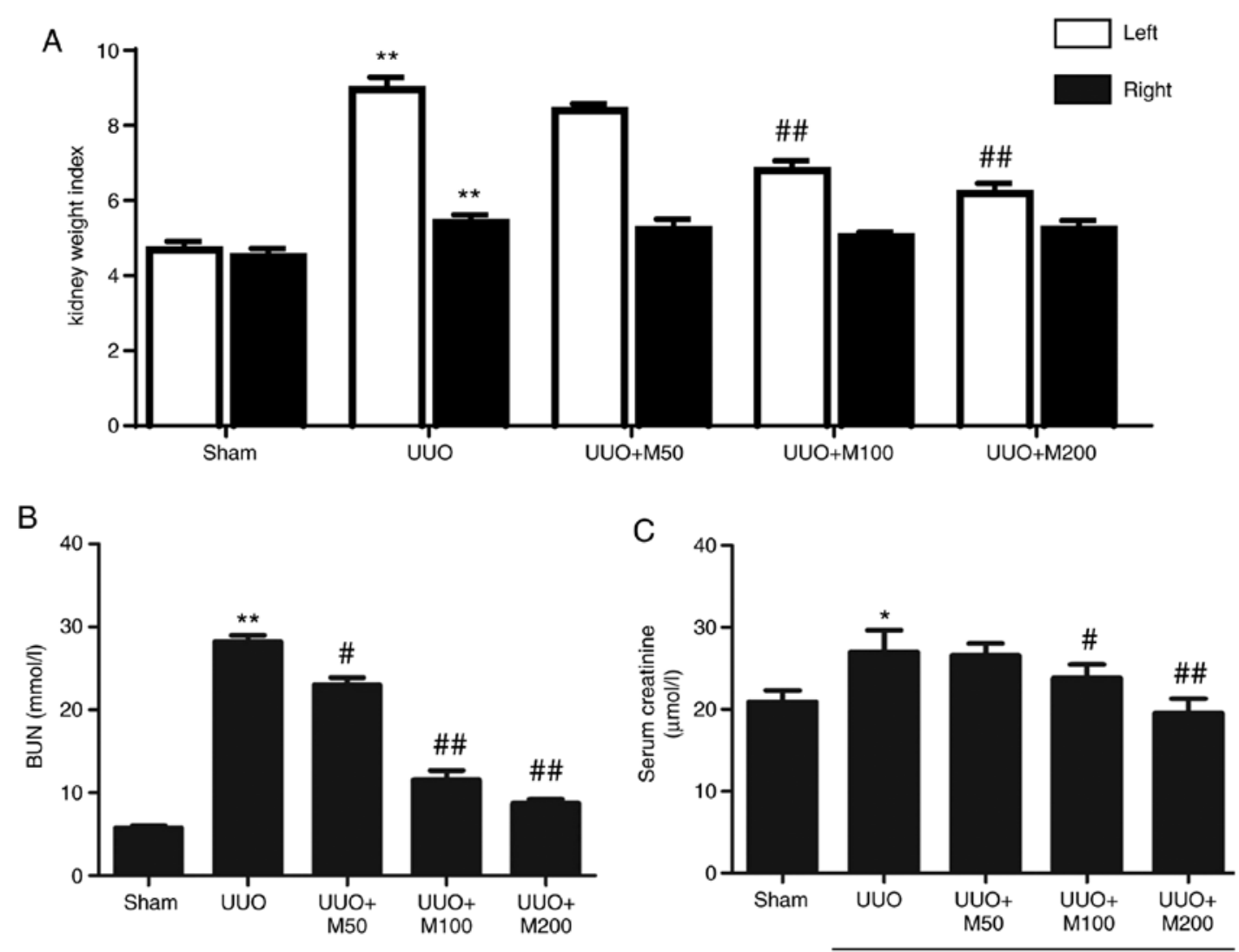

Figure 3. Effect of metformin on hypertrophy of kidney and renal function. Kidney weight index (mg/g) was expressed as the ratio of final kidney weight (mg) to final body weight (g). (A) Statistical analysis of kidney weight index. (B) Statistical analysis of BUN. (C) Statistical analysis of serum creatinine. All data are presented as the mean \pm SEM. $n=6 .{ }^{*} \mathrm{P}<0.05,{ }^{* *} \mathrm{P}<0.01$ vs. sham group; ${ }^{~} \mathrm{P}<0.05,{ }^{\# \#} \mathrm{P}<0.01$ vs. UUO group. Sham, Sham-operated group; UUO, unilateral ureteral obstruction group; UUO + M50, UUO rats treated with low-dose metformin, $50 \mathrm{mg} / \mathrm{kg} / \mathrm{day}$; UUO + M100, UUO rats treated with intermediate-dose metformin, $100 \mathrm{mg} / \mathrm{kg} / \mathrm{day}$; UUO + M200, UUO rats treated with high-dose metformin, $200 \mathrm{mg} / \mathrm{kg} / \mathrm{day}$; BUN, blood urea nitrogen.
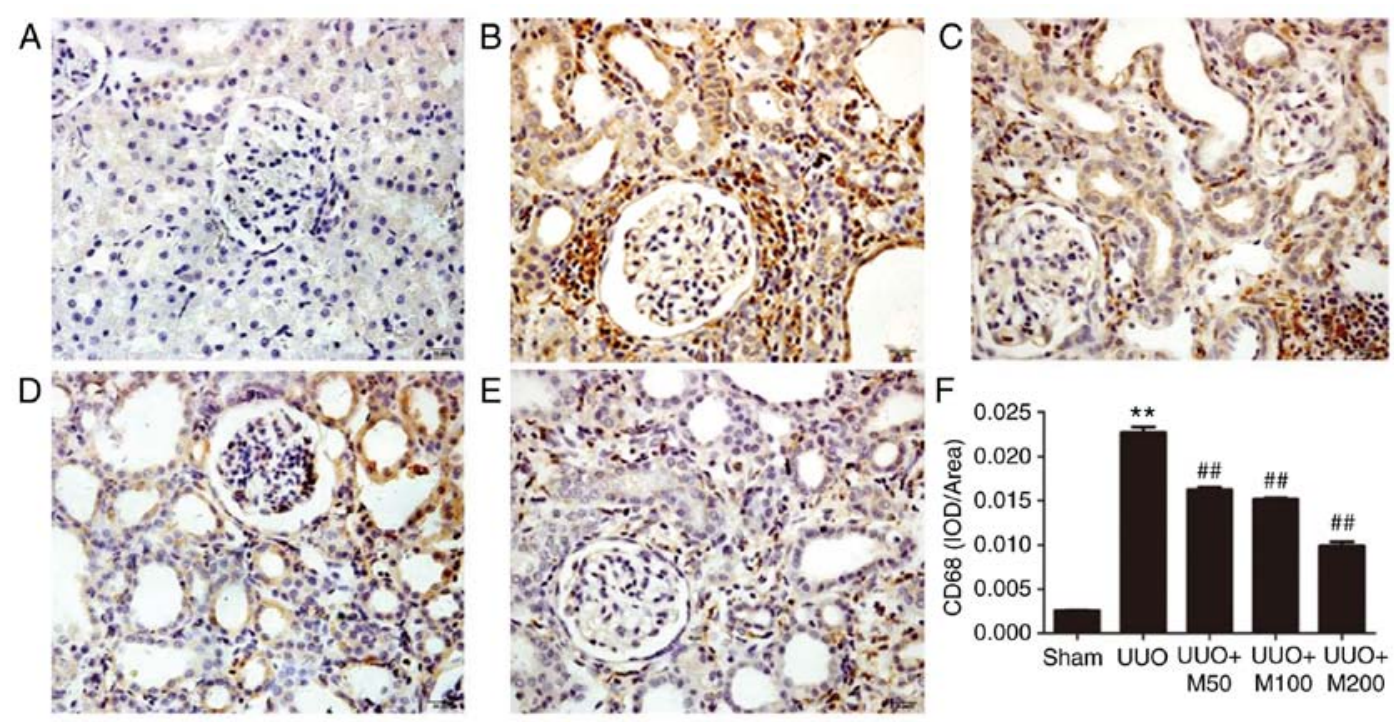

Figure 4. Effect of metformin on inflammation of obstructed kidney. (A) Sham operation. (B) UUO rats. (C) UUO rats treated with low-concentration of metformin, $50 \mathrm{mg} / \mathrm{kg} / \mathrm{day}$. (D) UUO rats treated with medium-concentration of metformin, $100 \mathrm{mg} / \mathrm{kg} / \mathrm{day}$. (E) UUO rats treated with high-concentration of metformin, $200 \mathrm{mg} / \mathrm{kg} /$ day. (F) Statistical analysis of CD68 expression. Magnification, $x 400$. All data are presented as the mean $\pm S E M$. $n=6$. ${ }^{* *} \mathrm{P}<0.01 \mathrm{vs.} \mathrm{sham}$ group; ${ }^{\# \#} \mathrm{P}<0.01$ vs. UUO group. UUO, unilateral ureteral obstruction; IOD, integral optical density.

Effects of metformin on the activity of the mTOR/p70S6K pathway in the kidneys of UUO rats. Deptor is an mTOR endogenous antagonist and metformin can upregulate Deptor expression. Therefore, the activation of the $\mathrm{mTOR} / \mathrm{p} 70 \mathrm{~S} 6 \mathrm{~K}$ signaling pathway was examined in renal tissues of UUO rats. The western blotting results suggested that there was 

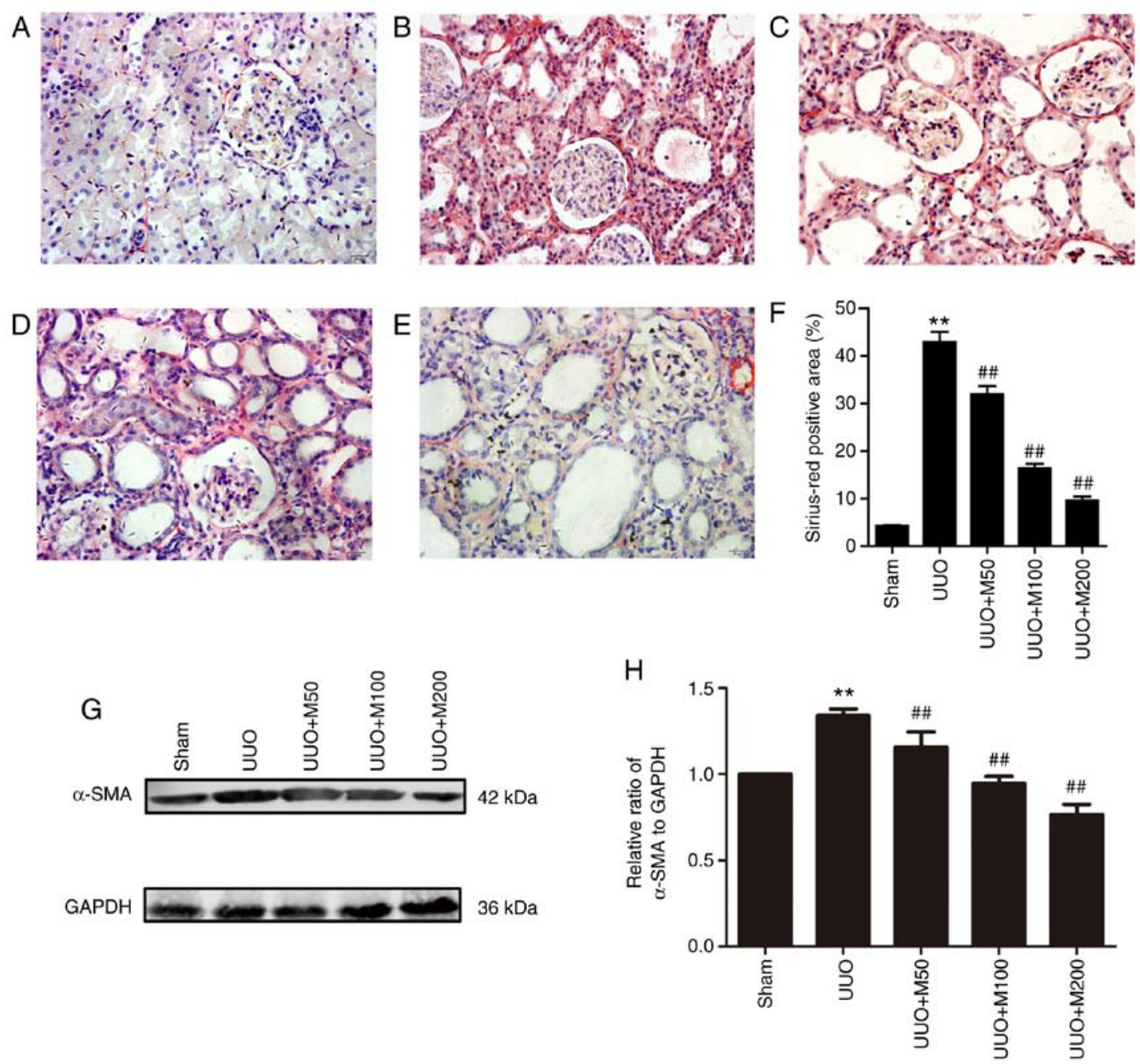

Figure 5. Effect of metformin on collagen expression and $\alpha$-SMA in obstructed kidney. (A) Sham operation. (B) UUO rats. (C) UUO rats treated with low-concentration metformin, $50 \mathrm{mg} / \mathrm{kg} / \mathrm{day}$. (D) UUO rats treated with medium-concentration metformin, $100 \mathrm{mg} / \mathrm{kg} / \mathrm{day}$. (E) UUO rats treated with high-concentration metformin, $200 \mathrm{mg} / \mathrm{kg} /$ day. (F) Statistical analysis of Sirius-Red positive area. Magnification, $x 400$. $\mathrm{n}=6$. (G) Protein expression level of $\alpha$-SMA in the kidneys of rats was analyzed by western blotting. (H) Statistical analysis of $\alpha$-SMA expression level. $n=3$. All data are presented as the mean \pm SEM. ${ }^{* *} \mathrm{P}<0.01$ vs. sham group; ${ }^{\# \#} \mathrm{P}<0.01$ vs. UUO group. UUO, unilateral ureteral obstruction; $\alpha$-SMA, $\alpha$-smooth muscle actin.

no significant difference in the expression of total mTOR (T-mTOR) and total p70S6K (T-p70S6K) in the kidneys of each group. The protein expression levels of p-mTOR and p-p70S6K in the kidneys of sham rats were almost absent. Compared with the sham group, the protein expression levels of p-mTOR and p-p70S6K in the kidneys of UUO rats were significantly increased, however metformin significantly decreased the phosphorylation levels of mTOR and p70S6K in the kidney of UUO rats, but only low dose of metformin had no significant difference (Fig. 7A-D).

\section{Discussion}

RIF is the most important factor determining the renal outcomes and clinical course of kidney diseases. In the present study, the expression level of Deptor was progressively downregulated over time in UUO rats. Moreover, the expression of collagen was found to be negatively correlated with the expression of Deptor in renal tissues of UUO rats. However, metformin significantly upregulated the protein expression level of Deptor, and downregulated the protein expression levels of p-mTOR and p-p70S6K, and three doses of metformin nearly reversed the changes stated above, except for the level of p-mTOR in low dose of metformin group.

Deptor, an endogenous mTOR inhibitory factor discovered by Peterson et al (10), is involved in the regulation of cell proliferation, survival, transdifferentiation and autophagy. Bruneau et al (11) reported that Deptor serves a key role in endogenous mechanisms of anti-inflammation and pro-resolution by regulating the endothelial cell-specific expression of chemokines and adhesion molecules, leukocyte-endothelial adhesion, and endothelial migratory responses. Recent studies found that Deptor serves an important role in various human diseases including kidney diseases $(12,24,25)$. Transforming growth factor beta 1 (TGF- $\beta 1$ ) serves an important role in the pathogenesis of renal fibrosis. TGF- $\beta 1$ can mediate chronic inflammation, myofibroblast activation and may promote ECM accumulation. It has been reported that TGF- $\beta 1$ can increase the activity of mTOR and promote collagen secretion by down-regulating Deptor expression $(12,15)$. The present study identified gradually increasing collagen deposition in the kidneys of rats in the UUO group in a time-dependent 

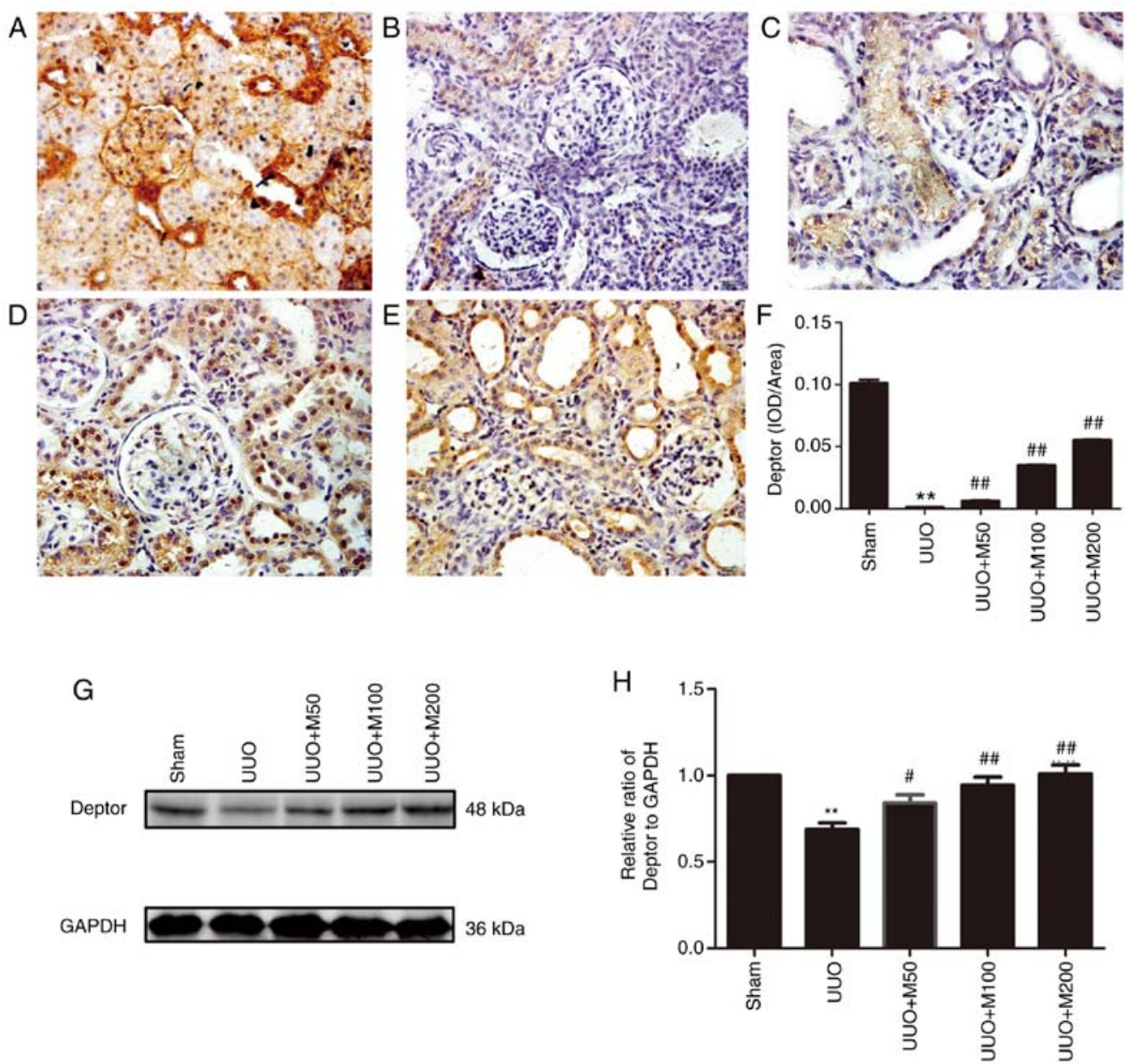

Figure 6. Effect of metformin on the expression of Deptor in obstructed kidneys analyzed by immunohistochemistry. Deptor expression in kidneys was investigated in various groups. (A) Sham operation. (B) UUO rats. (C) UUO rats treated with low-concentration metformin, $50 \mathrm{mg} / \mathrm{kg} / \mathrm{day}$. (D) UUO rats treated with medium-concentration metformin, $100 \mathrm{mg} / \mathrm{kg} /$ day. (E) UUO rats treated with high-concentration metformin, $200 \mathrm{mg} / \mathrm{kg} / \mathrm{day}$. (F) Statistical analysis of Deptor immunohistochemistry-positive area. $n=6$. (G) Protein expression of Deptor in the kidneys of rats was analyzed by western blotting. (H) Statistical analysis of Deptor expression level. Magnification, $x 400 . n=3$. All data are presented as the mean $\pm \mathrm{SEM} .{ }^{* *} \mathrm{P}<0.01$ vs. sham group; ${ }^{\# P} \mathrm{P}<0.05$, ${ }^{\# \#} \mathrm{P}<0.01$ vs. UUO group. UUO, unilateral ureteral obstruction; Deptor, DEP domain-containing mTOR interacting protein; IOD, integral optical density.

A

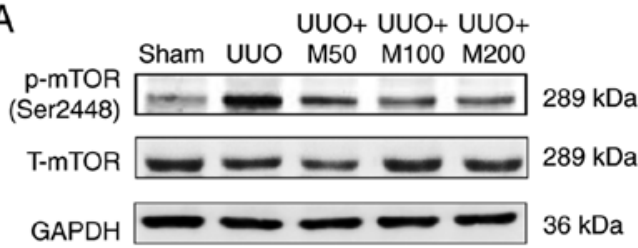

B

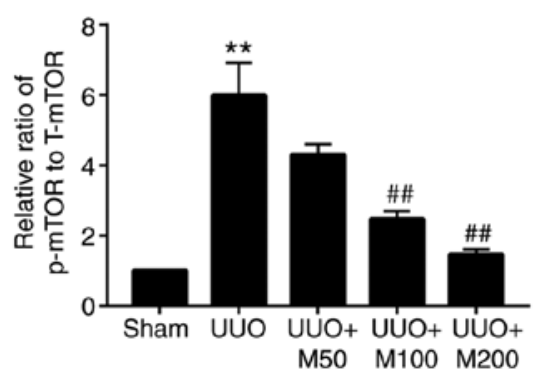

C

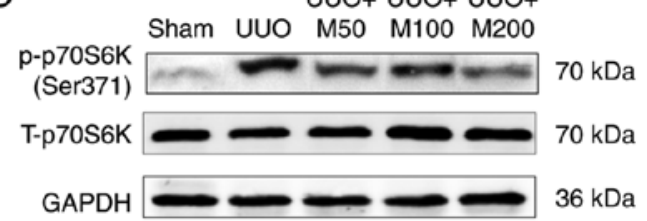

D

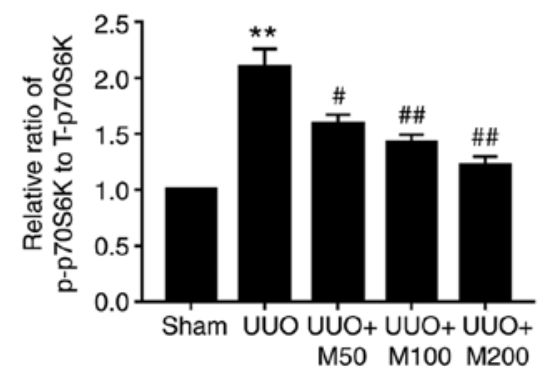

Figure 7. Effect of metformin on the mTOR/p70S6K pathway in obstructed kidneys. Western blot and quantitative analysis of the levels of p-mTOR (Ser2448) and p- p70S6K (Ser371) was performed following normalization to T-mTOR and T-p70S6K, respectively. GAPDH was used as an internal control. (A) p-mTOR levels were detected in rat kidneys by western blotting. (B) Statistical analysis of the levels of p-mTOR. (C) p-p70S6K levels were detected in rat kidneys by western blotting. (D) Statistical analysis of the levels of p-p70S6K. All data are presented as the mean \pm SEM. $n=3$. ${ }^{* *} \mathrm{P}<0.01$ vs. sham group; ${ }^{*} \mathrm{P}<0.05$, ${ }^{\# \#} \mathrm{P}<0.01$ vs. UUO group. Sham, Sham-operated group; UUO, unilateral ureteral obstruction group; UUO + M50, UUO rats treated with low-dose metformin, $50 \mathrm{mg} / \mathrm{kg} / \mathrm{day} ; \mathrm{UUO}+\mathrm{M} 100, \mathrm{UUO}$ rats treated with intermediate-dose metformin, $100 \mathrm{mg} / \mathrm{kg} / \mathrm{day} ; \mathrm{UUO}+\mathrm{M} 200, \mathrm{UUO}$ rats treated with high-dose metformin, $200 \mathrm{mg} / \mathrm{kg} /$ day; p-, phosphorylated; T-, total; p70S6K, ribosomal protein S6 kinase. 
manner, whereas the expression of Deptor in UUO kidneys decreased over time, indicating that Deptor may be involved in the development of the pathogenesis of renal fibrosis.

Metformin is the most widely used first-line biguanide drug in the treatment of type 2 diabetes. Metformin, in addition to its hypoglycemic effect, can also reduce cardiovascular complications in diabetic patients (26). Additionally, a previous study showed that metformin has antifibrotic activity (18). In animal models of non-alcoholic steatohepatitis, metformin could reduce the inflammatory response to prevent hepatic fibrosis (27). The present study found that metformin reduced the left renal mass index, Scr and BUN levels in UUO rat. Sirius-Red staining showed that metformin treatment also reduced the collagen area in renal tubules and interstitial regions of UUO rats. The present results suggested that metformin may significantly alleviate UUO-induced RIF and improve renal function.

Excessive deposition of ECM is one of the most important features of RIF. $\alpha-\mathrm{SMA}^{+}$myofibroblast can secret ECM components such as collagen. In the present study, the protein level of $\alpha$-SMA was upregulated in the kidney of UUO rats, suggesting an accumulation of myofibroblasts in the renal tissue of rats following UUO. The three concentrations of metformin tested in the present study were able to significantly reduce $\alpha$-SMA protein level and inhibit the formation of myofibroblast. In addition, Sirius-Red staining suggested that metformin reduced the deposition of collagen in the kidneys of UUO rats.

CD68 is an important marker of macrophages, and several previous studies demonstrated that macrophage infiltration promotes the development of RIF. Previous studies reported that macrophages could release inflammatory mediators such as interleukin- $\beta$ (IL- $\beta$ ) and a large number of cytokines to promote the expression of adhesion factors, increasing the accumulation of inflammatory factors in the inflammatory site, thus aggravating the inflammatory response (28). In addition, macrophages can also transform into myofibroblasts (MMT) to promote the synthesis of ECM, thus leading to RIF (29). The present results suggested that the expression of CD68 protein was increased in the kidney of UUO rats, and metformin treatment effectively inhibited its expression, indicating that metformin decreased the infiltration of macrophages, and reduced the inflammatory response and the MMT process, thereby delaying the progression of renal fibrosis.

Accumulating evidence demonstrated the detrimental effects of the mTOR/p70S6K pathway in renal fibrosis. In response to various stimuli, mTOR can be activated through phosphorylation, and the activated p-mTOR is involved in the regulation of protein synthesis by inducing the phosphorylation of p70S6K, which can phosphorylate the ribosomal protein S6 that is involved in protein translation $(30,31)$. Previous animal studies demonstrated that mTOR is highly activated in kidneys of diabetic nephropathy rats, but rapamycin could ameliorate glomerular hypertrophy, reducing proinflammatory cytokines expression, and relieving renal interstitial inflammation and fibrosis (32). In addition, rapamycin reduced macrophage infiltration in obstructed kidneys, reversed EMT progression and alleviated RIF (33). The present study suggested that the mTOR/p70S6K signaling pathway was inactive in kidneys from the sham group. However, the protein expression levels of p-mTOR and p-p70S6K were significantly upregulated following UUO via the activation of the mTOR/p70S6K signaling pathway in the kidneys of UUO rats. Administration of metformin for 14 days effectively inhibited the activation of the mTOR/p70S6K pathway induced by UUO.

Several reports have demonstrated that activation of adenosine monophosphate-activated protein kinase (AMPK) improves renal function in experimental models of acute kidney injury (AKI) (34) and CKD (35). The effects of metformin are mainly driven by the activation of AMPK, which regulates downstream signaling pathways (36). It was reported that AMPK also affects proteasomal degradation, and AMPK activation by metformin leads to an inhibition of proteasomal function (37). Evidences show that the protein levels of Deptor are known to be regulated by a proteasome-mediated proteolytic degradation (10). Previous study has reported that metformin increases the protein levels of Deptor via suppression of proteasome activity in an AMPK-dependent manner (17). In the present study, the expression of Deptor was significantly decreased in the kidneys of UUO rats, and metformin treatment efficiently increased the expression level of this protein. Therefore, we infer that activation of AMPK by metformin could inhibit the activity of proteasome, and subsequently upregulate the expression of Deptor, which leads to anti-fibrotic effect on rat renal fibrosis by suppressing mTOR signaling.

In conclusion, the present study suggested that metformin ameliorated the impaired renal function in a model of UUO-induced renal fibrosis, and the mechanism mediating this effect may be associated with the upregulation of Deptor expression level and the inhibition of the mTOR/p70S6K signaling pathway. Therefore, in addition to the anti-diabetic therapeutic potential of metformin, this drug may also be considered as a potential therapeutic for the treatment of renal fibrosis.

\section{Acknowledgements}

Not applicable.

\section{Funding}

The present study was supported by a grant from the Natural Science Foundation of Jiangsu Province (grant no. BK20161179).

\section{Availability of data and materials}

The datasets used and/or analyzed during the current study are available from the corresponding author on reasonable request.

\section{Authors' contributions}

AZX, YXW and YL conceived and designed the study. YL, LHL and YW performed the experiments. AZX and YXW wrote the manuscript. YXP and SZ analyzed the data. All authors read and approved the manuscript and agreed to be accountable for all aspects of the research in ensuring that the accuracy or integrity of any part of the work are appropriately investigated and resolved. 


\section{Ethics approval and consent to participate}

All animal experiments were approved by the Animal Ethics Committee of Xuzhou Medical University.

\section{Patient consent for publication}

Not applicable.

\section{Competing interests}

The authors declare that they have no competing interests.

\section{References}

1. Bai J, Hao J, Zhang X, Cui H, Han J and Cao N: Netrin-1 attenuates the progression of renal dysfunction by blocking endothelial-to-mesenchymal transition in the 5/6 nephrectomy rat model. BMC Nephrol 17: 47, 2016.

2. Zeisberg M and Neilson EG: Mechanisms of tubulointerstitial fibrosis. J Am Soc Nephrol 21: 1819-1834, 2010.

3. Rostaing L and Kamar N: mTOR inhibitor/proliferation signal inhibitors: Entering or leaving the field? J Nephrol 23: 133-142, 2010

4. Deblon N, Bourgoin L, Veyrat-Durebex C, Peyrou M, Vinciguerra M, Caillon A, Maeder C, Fournier M, Montet X, Rohner-Jeanrenaud F and Foti M: Chronic mTOR inhibition by rapamycin induces muscle insulin resistance despite weight loss in rats. Br J Pharmacol 165: 2325-2340, 2012.

5. Kim D, Cheng GZ, Lindsley CW, Yang H and Cheng JQ: Targeting the phosphatidylinositol-3 kinase/Akt pathway for the treatment of cancer. Curr Opin Investig Drugs 6: 1250-1258, 2005.

6. Takenaka T, Inoue T, Miyazaki T, Kobori H, Nishiyama A, Ishii N, Hayashi M and Suzuki H: Klotho ameliorates medullary fibrosis and pressure natriuresis in hypertensive rat kidneys. Hypertension 72: 1151-1159, 2018.

7. Chen G, Chen H, Wang C, Peng Y, Sun L, Liu H and Liu F: Rapamycin ameliorates kidney fibrosis by inhibiting the activation of mTOR signaling in interstitial macrophages and myofibroblasts. PLoS One 7: e33626, 2012.

8. Aboudehen K, Farahani S, Kanchwala M, Chan SC, Avdulov S, Mickelson A, Lee D, Gearhart MD, Patel V, Xing C and Igarashi P: Long noncoding RNA Hoxb3os is dysregulated in autosomal dominant polycystic kidney disease and regulates mTOR signaling. J Biol Chem 293: 9388-9398, 2018.

9. Raman A, Parnell SC, Zhang Y, Reif GA, Dai Y, Khanna A, DanielE, White C, Vivian JL and Wallace DP: Periostin overexpression in collecting ducts accelerates renal cyst growth and fibrosis in polycystic kidney disease. Am J Physiol Renal Physiol 315: F1695- F1707, 2018.

10. Peterson TR, Laplante M, Thoreen CC, Sancak Y, Kang SA, Kuehl WM, Gray NS and Sabatini DM: DEPTOR is an mTOR inhibitor frequently overexpressed in multiple myeloma cells and required for their survival. Cell 137: 873-886, 2009.

11. Bruneau S, Nakayama H, Woda CB, Flynn EA and Briscoe DM: DEPTOR regulates vascular endothelial cell activation and proinflammatory and angiogenic responses. Blood 122: 1833-1842,2013.

12. Das F, Ghosh-Choudhury N, Bera A, Dey N, Abboud HE, Kasinath BS and Choudhury GG: Transforming growth factor $\beta$ integrates Smad 3 to mechanistic target of rapamycin complexes to arrest deptor abundance for glomerular mesangial cell hypertrophy. J Biol Chem 288: 7756-7768, 2013.

13. Das F, Ghosh-Choudhury N, Lee DY, Gorin Y, Kasinath BS and Choudhury GG: Akt2 causes TGF $\beta$-induced deptor downregulation facilitating mTOR to drive podocyte hypertrophy and matrix protein expression. PLoS One 13: e0207285, 2018.

14. Maity S, Bera A, Ghosh-Choudhury N, Das F, Kasinath BS and Choudhury GG: microRNA-181a downregulates deptor for TGF $\beta$-induced glomerular mesangial cell hypertrophy and matrix protein expression. Exp Cell Res 364: 5-15, 2018.

15. Das F, Bera A, Ghosh-Choudhury N, Abboud HE, Kasinath BS and Choudhury GG: TGF $\beta$-induced deptor suppression recruits mTORC1 and not mTORC 2 to enhance collagen I $(\alpha 2)$ gene expression. PLoS One 9: e109608, 2014.

16. Wang C, Dai H, Xiong Z, Song Q, Zou Z, Li M, Nie J, Bai X and Chen Z: Loss of DEPTOR in renal tubules protects against cisplatin-induced acute kidney injury. Cell Death Dis 9: 441, 2018.
17. Obara A, Fujita Y, Abudukadier A, Fukushima T, Oguri Y, Ogura M, Harashima S, Hosokawa $M$ and Inagaki N: DEPTOR-related mTOR suppression is involved in metformin's anti-cancer action in human liver cancer cells. Biochem Biophys Res Commun 460: 1047-1052, 2015.

18. Cavaglieri RC, Day RT, Feliers D and Abboud HE: Metformin prevents renal interstitial fibrosis in mice with unilateral ureteral obstruction. Mol Cell Endocrinol 412: 116-122, 2015.

19. Oda SS: Metformin protects against experimental acrylamide neuropathy in rats. Drug Dev Res 78: 349-359, 2017.

20. Montinaro V, Hevey K, Aventaggiato L, Fadden K, Esparza A, Chen A, Finbloom DS and Rifai A: Extrarenal cytokines modulate the glomerular response to $\operatorname{Ig} \mathrm{A}$ immune complexes. Kidney Int 42: 341-353, 1992.

21. Sun D, Liu CX, Ma YY and Zhang L: Protective effect of prostaglandin E1 on renal microvascular injury in rats of acute aristolochic acid nephropathy. Ren Fail 33: 225-232, 2011.

22. Chen YJ, Kong L, Tang ZZ, Zhang YM, Liu Y, Wang TY and Liu YW: Hesperetin ameliorates diabetic nephropathy in rats by activating Nrf2/ARE/glyoxalase 1 pathway. Biomed Pharmacother 111: 1166-1175, 2019.

23. Xia A, Xue Z, Li Y, Wang W, Xia J, Wei T, Cao J and Zhou W: Cardioprotective effect of betulinic Acid on myocardial ischemia reperfusion injury in rats. Evid Based Complement Alternat Med 2014: 573745, 2014.

24. Caron A, Baraboi ED, Laplante $M$ and Richard D: DEP domain-containing mTOR-interacting protein in the rat brain: Distribution of expression and potential implication. J Comp Neurol 523: 93-107, 2015.

25. Davies J, Zachariades E, Rogers-Broadway KR and Karteris E: Elucidating the role of DEPTOR in Alzheimer's disease. Int J Mol Med 34: 1195-1200, 2014.

26. Wang CP, Lorenzo C, Habib SL, Jo B and Espinoza SE: Differential effects of metformin on age related comorbidities in older men with type 2 diabetes. J Diabetes Complications 31: 679-686, 2017.

27. Kita Y, Takamura T, Misu H, Ota T, Kurita S, Takeshita Y, Uno M, Matsuzawa-Nagata N, Kato K, Ando H, et al: Metformin prevents and reverses inflammation in a non-diabetic mouse model of nonalcoholic steatohepatitis. PLoS One 7: e43056, 2012.

28. Guiteras R, Flaquer M and Cruzado JM: Macrophage in chronic kidney disease. Clin Kidney J 9: 765-771, 2016.

29. Meng XM, Wang S, Huang XR, Yang C, Xiao J, Zhang Y, To KF, Nikolic-Paterson DJ and Lan HY: Inflammatory macrophages can transdifferentiate into myofibroblasts during renal fibrosis. Cell Death Dis 7: e2495, 2016.

30. Kapoor V, Zaharieva MM, Das SN and Berger MR: Erufosine simultaneously induces apoptosis and autophagy by modulating the Akt-mTOR signaling pathway in oral squamous cell carcinoma. Cancer Lett 319: 39-48, 2012

31. Krause U, Bertrand L and Hue L: Control of p70 ribosomal protein S6 kinase and acetyl-CoA carboxylase by AMP-activated protein kinase and protein phosphatases in isolated hepatocytes. Eur J Biochem 269: 3751-3759, 2002.

32. Yang Y, Wang J, Qin L, Shou Z, Zhao J, Wang H, Chen Y and Chen J: Rapamycin prevents early steps of the development of diabetic nephropathy in rats. Am J Nephrol 27: 495-502, 2007.

33. Wu MJ, Wen MC, Chiu YT, Chiou YY, Shu KH and Tang MJ: Rapamycin attenuates unilateral ureteral obstruction-induced renal fibrosis. Kidney Int 69: 2029-2036, 2006.

34. Feng J, Li H, Zhang Y, Wang Q, Zhao S, Meng P and Li J: Mammalian STE20-like kinase 1 deletion alleviates renal ischaemia-reperfusion injury via modulating mitophagy and the AMPK-YAP signalling pathway. Cell Physiol Biochem 51: 2359-2376, 2018.

35. Zhou X, Muise ES, Haimbach R, Sebhat IK, Zhu Y, Liu F, Souza SC, Kan Y, Pinto S, Kelley DE and Hoek M: PAN-AMPK activation improves renal function in a rat model of progressive diabetic nephropathy. J Pharmacol Exp Ther 371: 45-55, 2019.

36. Brynildsen JK, Lee BG, Perron IJ, Jin S, Kim SF and Blendy JA: Activation of AMPK by metformin improves withdrawal signs precipitated by nicotine withdrawal. Proc Natl Acad Sci USA 115: 4282-4287, 2018.

37. Viana R, Aguado C, Esteban I, Moreno D, Viollet B, Knecht E and Sanz P: Role of AMP-activated protein kinase in autophagy and proteasome function. Biochem Biophys Res Commun 369: 964-968, 2008.

This work is licensed under a Creative Commons Attribution-NonCommercial-NoDerivatives 4.0 International (CC BY-NC-ND 4.0) License. 